\title{
CYCLIC VECTORS OF WEIGHTED SHIFTS ON $l^{p}$ SPACES
}

\author{
B. S. YADAV AND S. CHATTERJEE
}

\begin{abstract}
Various authors have studied the existence of cyclic vectors of weighted shifts on Banach spaces (Hilbert spaces). In this paper, the existence of cyclic vectors of weighted shifts on $l^{p}$ Banach spaces is exhibited under suitable conditions on the weight sequence.
\end{abstract}

1. Introduction. Let $B$ be a Banach space and let $T$ be an operator on $B$, i.e., a bounded linear transformation of $B$ into itself. A vector $x \in B$ is called a cyclic vector of $T$ if

$$
B=\bigvee_{n=0}^{\infty}\left\{T^{n} x\right\}
$$

the closed subspace spanned by $x, T x, T^{2} x, \ldots$ The existence of cyclic vectors of operators on a Banach space (Hilbert space) has been the subject of investigation by many authors; see, for example, Douglas, Shapiro and Shields [2], [3], Gellar [4], Herrero [6], Deddens, Gellar and Herrero [1], Nikolskii [8], [9] and Rabindranathan [10]. The object of this note is to exhibit the existence of cyclic vectors of weighted shifts on the Banach space $l^{p}, 1 \leqslant p<\infty$, under suitable restrictions on their weight sequences.

2. Let $l^{p}, 1<p<\infty$, be the Banach space of all complex sequences $x=$ $\left\{x_{0}, x_{1}, x_{2}, \ldots\right\}$ with the norm $\|x\|=\left(\sum_{m=0}^{\infty}\left|x_{m}\right|^{p}\right)^{1 / p}$. We shall denote by $q$ the conjugate index of $p$, i.e. the number determined by $1 / p+1 / q=1$. Let $\left\{w_{m}\right\}_{m=1}^{\infty}$ be a bounded sequence of nonzero complex numbers. The unique operator $T$ on $l^{p}$ defined by

$$
T\left\{x_{0}, x_{1}, x_{2}, \ldots\right\}=\left\{w_{1} x_{1}, w_{2} x_{2}, \ldots\right\}
$$

is called the unilateral (backward) weighted shift on $l^{p}$ with the weight sequence $\left\{w_{m}\right\}_{m=1}^{\infty}$. We may and shall assume, without any loss of generality, that the weights $w_{m}$ are positive real numbers [5].

We denote by $\left\{n_{i}\right\}_{i=1}^{\infty}$ a sequence of positive integers with

$$
\left(n_{i+1}-n_{i}\right) / n_{i}>\xi>1, \quad i=1,2, \ldots,
$$

and set

$$
r_{i}^{(k)}=\sum_{m>n_{i}}\left|x_{m} / x_{n_{i}}\right|^{k}, \quad k=1,2, \ldots
$$

Received by the editors February 3, 1979 and, in revised form, May 30, 1979.

AMS (MOS) subject classifications (1970). Primary 47B99.

Key words and phrases. $l^{p}$ Banach spaces, weighted shift operators, cyclic vectors. 
We shall say that the sequence $\left\{w_{m}\right\}_{m=1}^{\infty}$ is of ' $r$ th power variation' if

$$
\sum_{m=1}^{\infty}\left|w_{m}^{r}-w_{m+1}^{r}\right|<\infty
$$

THEOREM 1. Let $T$ be a unilateral weighted shift on $l^{p}$ with weight sequence $\left\{w_{m}\right\}_{m=1}^{\infty}$ of qth power variation and let

$$
\lambda=\lambda(k, n)=\sum_{m=0}^{\infty}\left(\frac{w_{m+k} \ldots w_{m+n}}{w_{k} \ldots w_{n}}\right)^{q}<\infty
$$

for all $k(\geqslant 2)$ and $n(\geqslant k)$. Then any vector $x=\left\{x_{m}\right\}_{m=0}^{\infty}$ in $l^{p}$, such that (i) $x_{n_{i}} \neq 0, i=1,2, \ldots$, and (ii) $r_{i}^{(p)}<\infty$ for sufficiently large $i$, is a cyclic vector of $l^{p}$.

REMARK. The conditions of the type (2) on the weight sequence $\left\{w_{m}\right\}_{m=1}^{\infty}$ and vectors $x$ satisfying the prescribed conditions have been found useful in many other contexts also; see, for example, Kerlin and Lambert [7] and Nikolskii [9].

Proof. Let $\left\{e_{m}\right\}_{m=0}^{\infty}$ be the standard basis for $l^{p}$ and let $M=\bigvee_{n=0}^{\infty}\left\{T^{n} x\right\}$. We first show that $e_{0} \in M$.

By definition of $T$, we have $T x=\left\{w_{1} x_{1}, w_{2} x_{2}, \ldots\right\}$, and induction on $n$ gives

$$
T^{n} x=\left\{w_{1} \ldots w_{n} x_{n}, w_{2} \ldots w_{n+1} x_{n+1}, \ldots\right\} \text {. }
$$

It follows that

$$
\begin{aligned}
&\left\|\frac{T^{n_{i}} x}{w_{1} \ldots w_{n_{i}} x_{n_{i}}}-e_{0}\right\|^{p}=\sum_{m=n_{i}+1}^{\infty}\left(\frac{w_{m-n_{i}+1} \ldots w_{m}}{w_{1} \ldots w_{n_{i}}}\right)^{p}\left|\frac{x_{m}}{x_{n_{i}}}\right|^{p} \\
& \leqslant\left(\sum_{m=n_{i}+1}^{\infty}\left(\frac{w_{m-n_{i}+1} \ldots w_{m}}{w_{1} \ldots w_{n_{i}}}\right) \mid \frac{x_{m}}{x_{n_{i}}}\right)^{p} \\
&=\left(\sum_{m=0}^{\infty}\left(\frac{w_{m+2} \ldots w_{m+n_{i}+1}}{w_{1} \ldots w_{n_{i}}}\right)\left|\frac{x_{m+n_{i}+1}}{x_{n_{i}}}\right|\right)^{p} \\
& \leqslant\left(\sum_{m=0}^{\infty}\left(\frac{w_{m+2} \ldots w_{m+n_{i}+1}}{w_{1} \ldots w_{n_{i}}}\right)^{q}\right)^{p / q} \sum_{m>n_{i}}\left|\frac{x_{m}}{x_{n_{i}}}\right|^{p} \quad \text { (by Hölder's inequality) } \\
&=\frac{1}{w_{1}^{p}} r_{i}^{(p)}\left(\sum_{m=0}^{\infty}\left(\frac{w_{m+2} \ldots w_{m+n_{i}}}{w_{2} \ldots w_{n_{i}}}\right)^{q} w_{m+n_{i}+1}^{q}\right)^{p / q} \quad \text { (by (1)) } \\
&=\frac{1}{w_{1}^{p}} r_{i}^{(p)}\left(\sum_{m=0}^{\infty} \sum_{j=0}^{m}\left(\frac{w_{j+2} \ldots w_{j+n_{i}}}{w_{2} \ldots w_{n_{i}}}\right)^{q}\left(w_{m+n_{i}+1}^{q}-w_{m+n_{i}+2}^{q}\right)\right)^{p / q}
\end{aligned}
$$

(by Abel's transformation [11])

$\leqslant \frac{1}{w_{1}^{p}} r_{i}^{(p)} \lambda^{p / q}\left(\sum_{m=0}^{\infty}\left(w_{m+n_{i}+1}^{q}-w_{m+n_{i}+2}^{q}\right)\right)^{p / q}$

$\leqslant \frac{1}{w_{1}^{p}} r_{i}^{(p)} \lambda^{p / q}\left(\sum_{m>n_{i}}\left|w_{m}^{q}-w_{m+1}^{q}\right|\right)^{p / q}$

$\rightarrow 0$ as $i \rightarrow \infty$. 
Thus, $e_{0} \in M$.

It is immediate that, for each $n, y_{n}=T^{n} x-w_{1} \ldots w_{n} x_{n} e_{0}$ is in $M$. Proceeding as above, we get

$$
\begin{aligned}
& \left\|\frac{y_{n_{i+1}-1}}{w_{2} \ldots w_{n_{i+1}} x_{n_{i+1}}}-e_{1}\right\|^{p} \\
& \quad=\sum_{m=n_{i+1}+1}^{\infty}\left(\frac{w_{m-n_{i+1}+2} \cdots w_{m}}{w_{2} \cdots w_{n_{i+1}}}\right)^{p}\left|\frac{x_{m}}{x_{n_{i+1}}}\right|^{p} \\
& \leqslant \frac{1}{w_{2}^{p}} r_{i+1}^{(p)} \lambda^{p / q}\left(\sum_{m>n_{i+1}}\left|w_{m}^{q}-w_{m+1}^{q}\right|\right)^{p / q} \\
& \rightarrow 0 \text { as } i \rightarrow \infty .
\end{aligned}
$$

This gives $e_{1} \in M$. It is clear that we can continue in this manner to exhibit that $e_{m} \in M$ for all $m$. The theorem is proved.

In our next theorem, we show that the condition of $q$ th power variation on the weight sequence $\left\{w_{m}\right\}_{m=1}^{\infty}$ can be removed provided that the condition of eventual boundedness of the sequence $\left\{r_{i}\right\}_{i=1}^{\infty}$ is replaced by a more stringent condition.

THEOREM 2. Let $T$ be a unilateral weighted shift on $l^{p}$ with weight sequence $\left\{w_{m}\right\}_{m=1}^{\infty}$. If $(2)$ holds for all $k(\geqslant 1)$ and $n(\geqslant k)$, then any vector $x=\left\{x_{m}\right\}_{m=0}^{\infty}$ in $l^{p}$ such that (i) $x_{n_{i}} \neq 0, i=1,2, \ldots$, and (ii) $\sum_{i} r_{i}^{(p)}<\infty$, is a cyclic vector for $l^{p}$.

Proof. We have

$$
\begin{aligned}
\left\|\frac{T^{n_{i}} x}{w_{1} \ldots w_{n_{i}} x_{n_{i}}}-e_{0}\right\|^{p} & =\sum_{m=n_{i}+1}^{\infty}\left(\frac{w_{m-n_{i}+1} \ldots w_{m}}{w_{1} \ldots w_{n_{i}}}\right)^{p}\left|\frac{x_{m}}{x_{n_{i}}}\right|^{p} \\
& \leqslant\left(\sum_{m=0}^{\infty}\left(\frac{w_{m+2} \ldots w_{m+n_{i}+1}}{w_{1} \ldots w_{n_{i}}}\right) \mid \frac{x_{m+n_{i}+1}}{x_{n_{i}}}\right)^{p} \\
& \leqslant\left(\sum_{m=0}^{\infty}\left(\frac{w_{m+2} \ldots w_{m+n_{i}+1}}{w_{1} \ldots w_{n_{i}}}\right)^{q}\right)^{p / q} \sum_{m>n_{i}}\left|\frac{x_{m}}{x_{n_{i}}}\right|^{p}
\end{aligned}
$$

(by Hölder's inequality)

$$
\leqslant \lambda^{p / q} r_{i}^{(p)} \rightarrow 0 \text { as } i \rightarrow \infty .
$$

A computation analogous to that given in the proof of Theorem 1 shows that $e_{m} \in \bigvee_{n=0}^{\infty}\left\{T^{n} x\right\}$ for all $m$. This completes our proof.

In the case $p=1$, we have the following two theorems corresponding to Theorems 1 and 2 .

THEOREM 3. Let $T$ be a unilateral weighted shift on $l^{1}$ with weight sequence $\left\{w_{m}\right\}_{m=1}^{\infty}$ in $l^{1}$, and let

$$
\delta=\sup _{p, m, n} \frac{w_{p+m} \cdots w_{p+n}}{w_{m} \cdots w_{n}}<\infty
$$


for all $p(\geqslant 0), m(\geqslant 2)$ and $n(\geqslant m)$. Then any vector $x=\left\{x_{m}\right\}_{m=0}^{\infty}$ in $l^{1}$, such that (i) $x_{n_{i}} \neq 0, i=1,2, \ldots$, and (ii) $r_{i}^{(1)}<\infty$ for sufficiently large $i$, is a cyclic vector for $l^{1}$.

THEOREM 4. Let $T$ be a unilateral weighted shift on $l^{1}$ with weight sequence $\left\{w_{m}\right\}_{m=1}^{\infty}$. If $(3)$ holds for all $p(\geqslant 0), m(\geqslant 1)$ and $n(\geqslant m)$, then any vector $x=\left\{x_{m}\right\}_{m=0}^{\infty}$ in $l^{1}$ such that (i) $x_{n_{i}} \neq 0, i=1,2, \ldots$, and (ii) $\sum_{i} r_{i}^{(1)}<\infty$, is a cyclic vector for $l^{1}$.

We shall only sketch the proofs of these theorems. In case of Theorem 3, following the proof of Theorem 1, it suffices to observe that

$$
\begin{aligned}
\left\|\frac{T^{n_{i}} x}{w_{1} \ldots w_{n_{i}} x_{n_{i}}}-e_{0}\right\| & =\sum_{m=n_{i}+1}^{\infty}\left(\frac{w_{m-n_{i}+1} \ldots w_{m}}{w_{1} \ldots w_{n_{i}}}\right)\left|\frac{x_{m}}{x_{n_{i}}}\right| \\
& =\sum_{m=0}^{\infty}\left(\frac{w_{m+2} \ldots w_{m+n_{i}+1}}{w_{1} \ldots w_{n_{i}}}\right)\left|\frac{x_{m+n_{i}+1}}{x_{n_{i}}}\right| \\
& =\frac{w_{n_{i}+1}}{w_{1}} \sum_{m=0}^{\infty}\left(\frac{w_{m+2} \ldots w_{m+n_{i}+1}}{w_{2} \ldots w_{n_{i}+1}}\right)\left|\frac{x_{m+n_{i}+1}}{x_{n_{i}}}\right| \\
& \leqslant \frac{w_{n_{i}+1}}{w_{1}} \delta \sum_{m>n_{i}}\left|\frac{x_{m}}{x_{n_{i}}}\right| \\
& =\frac{\delta r_{i}^{(1)}}{w_{1}} w_{n_{i}+1} \rightarrow 0 \text { as } i \rightarrow \infty,
\end{aligned}
$$

for $w_{n_{i}+1} \rightarrow 0$ as $i \rightarrow \infty$ and $r_{i}^{(1)}$ is finite for sufficiently large $i$.

For the proof of Theorem 4, we see that

$$
\begin{aligned}
& \left\|\frac{T^{n_{i} x}}{w_{1} \ldots w_{n_{i}} x_{n_{i}}}-e_{0}\right\| \\
& \quad=\sum_{m=0}^{\infty}\left(\frac{w_{m+2} \cdots w_{m+n_{i}+1}}{w_{1} \ldots w_{n_{i}}}\right)\left|\frac{x_{m+n_{i}+1}}{x_{n_{i}}}\right| \\
& \leqslant \delta \sum_{m>n_{i}}\left|\frac{x_{m}}{x_{n_{i}}}\right|=\delta r_{i}^{(1)} \rightarrow 0 \text { as } i \rightarrow \infty .
\end{aligned}
$$

Our last result investigates conditions under which a vector $x=\left\{x_{m}\right\}_{m=0}^{\infty}$ with $\left\{\left|x_{m}\right|\right\}_{m=0}^{\infty}$ monotonically decreasing serves as a cyclic vector for a weighted shift on $l^{p}, 1<p<\infty$.

THEOREM 5. Let $T$ be a unilateral weighted shift on $l^{p}$ with weight sequence $\left\{w_{m}\right\}_{m=1}^{\infty}$ of pth power variation. If for all $k(\geqslant 2)$ and $n(\geqslant k)$

$$
\lambda=\lambda(k, n)=\sum_{m=0}^{\infty}\left(\frac{w_{m+k} \ldots w_{m+n}}{w_{k} \ldots w_{n}}\right)^{p}<\infty,
$$

then any vector $x=\left\{x_{m}\right\}_{m=0}^{\infty}$ in $l^{p}$ such that $\left|x_{m}\right| \geqslant\left|x_{m+1}\right|>0, m=0,1,2, \ldots$, is a cyclic vector for $l^{p}$. 
Proof. Proceeding as in Theorem 1, we have

$$
\begin{aligned}
\left\|\frac{T^{n} x}{w_{1} \ldots w_{n} x_{n}}-e_{0}\right\|^{p} & =\sum_{m=n+1}^{\infty}\left(\frac{w_{m-n+1} \ldots w_{m}}{w_{1} \ldots w_{n}}\right)^{p}\left|\frac{x_{m}}{x_{n}}\right|^{p} \\
& \leqslant \sum_{m=0}^{\infty}\left(\frac{w_{m+2} \ldots w_{m+n+1}}{w_{1} \ldots w_{n}}\right)^{p} \\
& =\frac{1}{w_{1}^{p}} \sum_{m=0}^{\infty}\left(\frac{w_{m+2} \ldots w_{m+n}}{w_{2} \ldots w_{n}}\right)^{p} w_{m+n+1}^{p} \\
& =\frac{1}{w_{1}^{p}} \sum_{m=0}^{\infty} \sum_{j=0}^{m}\left(\frac{w_{j+2} \ldots w_{j+n}}{w_{2} \ldots w_{n}}\right)^{p}\left(w_{m+n+1}^{p}-w_{m+n+2}^{p}\right) \\
& \leqslant \frac{1}{w_{1}^{p}} \lambda \sum_{m=0}^{\infty} \mid w_{m+n+1}^{p}-w_{m+n+2}^{p} \quad \quad \text { (by Abel's transformation [11]) } \\
& =\frac{1}{w_{1}^{p}} \lambda \sum_{m>n}\left|w_{m}^{p}-w_{m+1}^{p}\right| \rightarrow 0 \quad \text { as } n \rightarrow \infty .
\end{aligned}
$$

This shows that $e_{0} \in \bigvee_{n=0}^{\infty}\left\{T^{n} x\right\}$. Now we follow exactly the line of argument as given in the proof of Theorem 1 and we are done.

The authors thank the referee for his suggestion to rewrite Theorems 3 and 4 in the present form.

\section{REFERENCES}

1. James A. Deddens, Ralph Gellar and Domingo A. Herrero, Commutants and cyclic vectors, Proc. Amer. Math. Soc. 43 (1974), 169-170.

2. R. G. Douglas, H. S. Shapiro and A. L. Shields, On cyclic vectors of the backward shift, Bull. Amer. Math. Soc. 73 (1967), 156-159.

3. Cyclic vectors and invariant subspaces for the backward shift operator, Ann. Inst. Fourier (Grenoble) 20 (1970), fasc. 1, 37-76.

4. R. Gellar, Cyclic vectors and parts of the spectrum of a weighted shift, Trans. Amer. Math. Soc. 146 (1969), 69-85.

5. P. R. Halmos, A Hilbert space problem book, Van Nostrand, Princeton, N. J., 1967.

6. D. A. Herrero, Eigenvectors and cyclic vectors for bilateral weighted shifts, Rev. Un. Mat. Argentina 26 (1972), 26-41.

7. Edward Kerlin and Alan Lambert, Strictly cyclic shifts on $l^{p}$, Acta Sci. Math. (Szeged) 35 (1973), 87-94.

8. N. K. Nikol'skii, The invariant subspaces of certain completely continuous operators, Vestnik Leningrad Univ. (7) 20 (1965), 68-77. (Russian)

9. Invariant subspaces of weighted shift operators, Mat. Sb. (N. S.) 74 (116) (1967), 172-190.

10. M. Rabindranathan, On cyclic vectors of weighted shifts, Proc. Amer. Math. Soc. 44 (1974), 293-299.

11. A. Zygmund, Trigonometric series, 2nd ed., Vol. I, Cambridge Univ. Press, New York, 1959.

Department of Mathematics, University of Delhi, Delhi-110007, India 